\title{
Nontrivial Solutions for a Class of Fractional Differential Equations with Integral Boundary Conditions and a Parameter in a Banach Space with Lattice
}

\author{
Xingqiu Zhang and Lin Wang \\ School of Mathematics, Liaocheng University, Liaocheng, Shandong 252059, China \\ Correspondence should be addressed to Xingqiu Zhang, zhxq197508@163.com
}

Received 17 September 2012; Accepted 12 December 2012

Academic Editor: Zhanbing Bai

Copyright (C) 2012 X. Zhang and L. Wang. This is an open access article distributed under the Creative Commons Attribution License, which permits unrestricted use, distribution, and reproduction in any medium, provided the original work is properly cited.

Existence of nontrivial solutions for the following fractional differential equation with integral boundary conditions $D_{0+}^{\alpha} u(t)+h(t) f(t, u(t))=0,0<t<1, u(0)=u^{\prime}(0)=u^{\prime \prime}(0)=0, u(1)=$ $\lambda \int_{0}^{\eta} u(s) \mathrm{d} s$ is investigated by using results for the computation of topological degree under the lattice structure, where $3<\alpha \leq 4,0<\eta \leq 1,0 \leq \lambda \eta^{\alpha} / \alpha<1, D_{0+}^{\alpha}$ is the standard Riemann-Liouville derivative. $h(t)$ is allowed to be singular at $t=0$ and $t=1$.

\section{Introduction}

Fractional differential equations have been of great interest for many researchers recently. It is caused both by the intensive development of the theory of fractional calculus itself and by the applications of such constructions in various fields of sciences and engineering such as control, porous media, electromagnetic, and other fields. For an extensive collection of such results, we refer the readers to the monographs by Samko et al. [1], Podlubny [2], and Kilbas et al. [3]. Recently, there are some papers dealing with the existence of solutions (or positive solutions) for nonlinear fractional differential equation by means of techniques of nonlinear analysis (fixed point theorems, Leray-Schauder theory, adomian decomposition method, lower and upper solution method, etc.); see [4-16].

As is well known, the first eigenvalue is a character of great significance for the linear operator. For some integer order differential equations, many authors have investigated the existence of positive and nontrivial solutions concerning the first eigenvalue corresponding to the relevant linear operators when the nonlinearities are sublinear, see [17-22] for reference. 
On the other hand, papers [23-26] obtained similar results to the sublinear case. The main discussion is based on the concepts of dual space, dual cone, and a constructed cone on them.

Recently, Xu et al. [27] and Bai [28] obtained the existence results of positive solutions for some fractional differential equations under the conditions with respect to the first eigenvalue corresponding to the relevant linear operators.

In two recent papers [29, 30], Sun and Liu established some results about the computation of the topological degree for nonlinear operators which are not cone mappings using the lattice structure.

Motivated by the above papers, by using results for the computation of topological degree under the lattice structure, we investigate the existence of nontrivial solutions for the following nonlinear fractional differential equations with integral boundary conditions:

$$
\begin{gathered}
D_{0+}^{\alpha} u(t)+h(t) f(t, u(t))=0, \quad 0<t<1, \\
u(0)=u^{\prime}(0)=u^{\prime \prime}(0)=0, \\
u(1)=\lambda \int_{0}^{\eta} u(s) \mathrm{d} s,
\end{gathered}
$$

where $3<\alpha \leq 4,0<\eta \leq 1,0 \leq \lambda \eta^{\alpha} / \alpha<1, D_{0+}^{\alpha}$ is the standard Riemann-Liouville derivative. In this paper, it is not required that nonlinearity $f(t, u) \geq 0$, for all $u \geq 0$. To the author's knowledge, few papers are available in the literature to study the existence of solutions for fractional differential equations with integral boundary conditions under the lattice structure. The method used in this paper is different from those in previous works.

This paper is organized as follows. In Section 2 corresponding Green's function for BVP (1.1) is derived and its properties are also discussed. The main results and their proof are presented in Section 3.

\section{Background Materials and Green's Function}

Let $E$ be a Banach space with a cone $P$. Then $E$ becomes an ordered Banach space under the partial ordering $\leq$ which is induced by $P . P$ is said to be normal if there exists a positive constant $N$ such that $\theta \leq x \leq y$ implies $\|x\| \leq N\|y\| . P$ is called solid if it contains interior points, that is, int $P \neq \emptyset$. $P$ is called total if $E=\overline{P-P}$. If $P$ is solid, then $P$ is total. For the concepts and the properties about the cone we refer to [31,32].

We call $E$ a lattice under the partial ordering $\leq \operatorname{if} \sup \{x, y\}$ and $\inf \{x, y\}$ exist for arbitrary $x, y \in E$. For $x \in E$, let $x^{+}=\sup \{x, \theta\}, x^{-}=\sup \{-x, \theta\}, x^{+}$and $x^{-}$are called the positive part and the negative part of $x$, respectively, and obviously $x=x^{+}-x^{-}$. Take $|x|=x^{+}+x^{-}$, then $|x| \in P$. One can refer to [33] for the definition and the properties of the lattice. Let $x_{+}=x^{+}, x_{-}=-x^{-}$as denoted in $[29,30]$. Then $x_{+} \in P, x_{-} \in-P$ and $x=x_{+}+x_{-}$.

Let $B: E \rightarrow E$ be a bounded linear operator. $B$ is said to be positive if $B(P) \rightarrow P$. In this case, $B$ is an increase operator, namely, for $x, y \in E, x \leq y$ implies $B x \leq B y$. Let $B: E \rightarrow E$ be a positively completely continuous operator, $r(B)$ a spectral radius of $B, B^{*}$ the conjugated operator of $B, P^{*}$ the conjugated cone of $P$. Since $P \subset E$ is a total cone, according to the famous 
Krein-Rutman theorem (see [34]), we infer that if $r(B) \neq 0$, then there exist $\bar{\varphi} \in P \backslash\{\theta\}$ and $g^{*} \in P^{*} \backslash\{\theta\}$ such that

$$
B \varphi=r(B) \varphi, \quad B^{*} g^{*}=r(B) g^{*} .
$$

For a given constant $\delta>0$, set

$$
P\left(g^{*}, \delta\right)=\left\{x \in P, g^{*}(x) \geq \delta\|x\|\right\},
$$

then $P\left(g^{*}, \delta\right)$ is also a cone in $E$.

Definition 2.1 (see [30]). Let $D \subset E$ and $F: D \rightarrow E$ a nonlinear operator. $F$ is said to be quasiadditive on lattice, if there exists $y_{0} \in E$ such that

$$
F x=F x_{+}+F x_{-}+y_{0}, \quad \forall x \in D
$$

Definition 2.2 (see [30]). Let $B$ be a positive linear operator. The operator $B$ is said to satisfy $H$ condition, if there exist $\bar{\varphi} \in P \backslash\{\theta\}, g^{*} \in P^{*} \backslash\{\theta\}$ such that (2.1) holds, and $B$ maps $P$ into $P\left(g^{*}, \delta\right)$.

Definition 2.3 (see [4]). The Riemann-Liouville fractional integral of order $\alpha>0$ of a function $y:(0, \infty) \rightarrow R$ is given by

$$
I_{0+}^{\alpha} y(t)=\frac{1}{\Gamma(\alpha)} \int_{0}^{t}(t-s)^{\alpha-1} y(s) \mathrm{d} s
$$

provided the right-hand side is pointwise defined on $(0, \infty)$.

Definition 2.4 (see [4]). The Riemann-Liouville fractional derivative of order $\alpha>0$ of a continuous function $y:(0, \infty) \rightarrow R$ is given by

$$
D_{0+}^{\alpha} y(t)=\frac{1}{\Gamma(n-\alpha)}\left(\frac{\mathrm{d}}{\mathrm{d} t}\right)^{n} \int_{0}^{t} \frac{y(s)}{(t-s)^{\alpha-n+1}} \mathrm{~d} s,
$$

where $n=[\alpha]+1,[\alpha]$ denotes the integer part of the number $\alpha$, provided that the right-hand side is pointwise defined on $(0, \infty)$.

Lemma 2.5 (see [29]). Let $P$ be a normal solid cone in $E$ and $A: E \rightarrow E$ completely continuous and quasiadditive on lattice. Suppose that the following conditions are satisfied:

(i) there exist a positive bounded linear operator $B_{1}, u^{*} \in P$ and $u_{1} \in P$, such that

$$
-u^{*} \leq A x \leq B_{1} x+u_{1}, \quad \forall x \in P
$$

(ii) there exist a positive bounded linear operator $B_{2}$ and $u_{2} \in P$, such that

$$
A x \geq B_{2} x-u_{2}, \quad \forall x \in(-P)
$$


(iii) $r\left(B_{1}\right)<1, r\left(B_{2}\right)<1$, where $r\left(B_{i}\right)$ is the spectral radius of $B_{i}(i=1,2)$. Then there exists $R_{0}>0$ such that for $R>R_{0}$, the topological degree $\operatorname{deg}\left(I-A, B_{R}, \theta\right)=1$.

Lemma 2.6 (see [29]). Let $P$ be a normal cone of $E$, and $A: E \rightarrow E$ a completely continuous operator. Suppose that there exist positive bounded linear operator $B_{0}$ and $u_{0} \in P$, such that

$$
|A x| \leq B_{0}|x|+u_{0}, \quad \forall x \in E .
$$

If $r\left(B_{0}\right)<1$, then there exists $R_{0}>0$ such that for $R>R_{0}$ the topological degree $\operatorname{deg}\left(I-A, B_{R}, \theta\right)=1$.

Lemma 2.7 (see [30]). Let $P$ be a solid cone in $E$ and $A: E \rightarrow E$ a completely continuous operator with $A=B F$, where $F$ is quasiadditive on lattice, and $B$ is a positive bounded linear operator satisfying $H$ condition. Suppose that

(i) there exist $a_{1}>r^{-1}(B)$ and $y_{1} \in P$ such that

$$
F x \geq a_{1} x-y_{1}, \quad \forall x \in P
$$

(ii) there exist $0<a_{2}<r^{-1}(B)$ and $y_{2} \in P$ such that

$$
F x \geq a_{2} x-y_{2}, \quad \forall x \in(-P)
$$

Then there exists $R_{0}>0$ such that for $R>R_{0}$ the topological degree $\operatorname{deg}\left(I-A, B_{R}, \theta\right)=0$.

Lemma 2.8 (see [30]). Let $\Omega \subset E$ be a bounded open set which contains $\theta$. Suppose that $A: \bar{\Omega} \rightarrow E$ is a completely continuous operator which has no fixed point on $\partial \Omega$. If

(i) there exists a positive bounded linear operator B such that

$$
|A x| \leq B_{0}|x|, \quad \forall x \in \partial \Omega
$$

(ii) $r\left(B_{0}\right) \leq 1$, then the topological degree $\operatorname{deg}(I-A, \Omega, \theta)=1$.

Lemma 2.9 (see [4]). Let $\alpha>0$. If one assumes $u \in C(0,1) \cap L(0,1)$, then the fractional differential equation

$$
D_{0+}^{\alpha} u(t)=0,
$$

has $u(t)=C_{1} t^{\alpha-1}+C_{2} t^{\alpha-2}+\cdots+C_{N} t^{\alpha-N}, C_{i} \in R, i=1,2, \ldots, N$, as unique solution, where $N$ is the smallest integer greater than or equal to $\alpha$.

Lemma 2.10 (see [4]). Assume that $u \in C(0,1) \cap L(0,1)$ with a fractional derivative of order $\alpha>0$ that belongs to $C(0,1) \cap L(0,1)$.

Then

$$
I_{0+}^{\alpha} D_{0+}^{\alpha} u(t)=u(t)+C_{1} t^{\alpha-1}+C_{2} t^{\alpha-2}+\cdots+C_{N} t^{\alpha-N},
$$

for some $C_{i} \in R, i=1,2, \ldots, N$, where $N$ is the smallest integer greater than or equal to $\alpha$. 
In the following, we present Green's function of the fractional differential equation boundary value problem.

Lemma 2.11. Given $y \in C[0,1]$, the problem

$$
\begin{gathered}
D_{0+}^{\alpha} u(t)+y(t)=0, \\
u(0)=u^{\prime}(0)=u^{\prime \prime}(0)=0, \\
u(1)=\lambda \int_{0}^{\eta} u(s) d s,
\end{gathered}
$$

where $0<t<1,3<\alpha \leq 4,0<\eta \leq 1,0 \leq \lambda \eta^{\alpha} / \alpha<1$ is equivalent to

$$
u(t)=\int_{0}^{1} G(t, s) y(s) d s,
$$

where

$$
G(t, s)= \begin{cases}\frac{t^{\alpha-1}(1-s)^{\alpha-1}-(\lambda / \alpha)(\eta-s)^{\alpha} t^{\alpha-1}-\left(1-(\lambda / \alpha) \eta^{\alpha}\right)(t-s)^{\alpha-1}}{p(0) \Gamma(\alpha)}, & 0 \leq s \leq t \leq 1, s \leq \eta ; \\ \frac{t^{\alpha-1}(1-s)^{\alpha-1}-\left(1-(\lambda / \alpha) \eta^{\alpha}\right)(t-s)^{\alpha-1}}{p(0) \Gamma(\alpha)}, & 0<\eta \leq s \leq t \leq 1 ; \\ \frac{t^{\alpha-1}(1-s)^{\alpha-1}-(\lambda / \alpha)(\eta-s)^{\alpha} t^{\alpha-1}}{p(0) \Gamma(\alpha)}, & 0 \leq t \leq s \leq \eta \leq 1 ; \\ \frac{t^{\alpha-1}(1-s)^{\alpha-1}}{p(0) \Gamma(\alpha)}, & 0 \leq t \leq s \leq 1, \eta \leq s .\end{cases}
$$

Here, $p(s):=1-\left(\lambda \eta^{\alpha} / \alpha\right)(1-s), G(t, s)$ is called the Green function of BVP (2.14). Obviously, $G(t, s)$ is continuous on $[0,1] \times[0,1]$.

Proof. We may apply Lemma 2.10 to reduce (2.14) to an equivalent integral equation

$$
u(t)=-I_{0+}^{\alpha} y(t)+C_{1} t^{\alpha-1}+C_{2} t^{\alpha-2}+C_{3} t^{\alpha-3}+C_{4} t^{\alpha-4},
$$

for some $C_{1}, C_{2}, C_{3}, C_{4} \in R$. Consequently, the general solution of (2.14) is

$$
u(t)=-\frac{1}{\Gamma(\alpha)} \int_{0}^{t}(t-s)^{\alpha-1} y(s) \mathrm{d} s+C_{1} t^{\alpha-1}+C_{2} t^{\alpha-2}+C_{3} t^{\alpha-3}+C_{4} t^{\alpha-4}
$$


By $u(0)=u^{\prime}(0)=u^{\prime \prime}(0)=0$, we get that $C_{2}=C_{3}=C_{4}=0$. On the other hand, boundary condition $u(1)=\lambda \int_{0}^{\eta} u(s) \mathrm{d} s$ combining with

$$
\begin{aligned}
u(1) & =-\int_{0}^{1} \frac{(1-s)^{\alpha-1}}{\Gamma(\alpha)} y(s) \mathrm{d} s+C_{1} \\
\int_{0}^{\eta} u(t) \mathrm{d} t & =-\frac{1}{\Gamma(\alpha)} \int_{0}^{\eta} \int_{0}^{t}(t-s)^{\alpha-1} y(s) \mathrm{d} s \mathrm{~d} t+C_{1} \int_{0}^{\eta} s^{\alpha-1} \mathrm{~d} s \\
& =-\frac{1}{\Gamma(\alpha)} \int_{0}^{\eta} \int_{s}^{\eta}(t-s)^{\alpha-1} y(s) \mathrm{d} t \mathrm{~d} s+C_{1} \int_{0}^{\eta} s^{\alpha-1} \mathrm{~d} s \\
& =-\frac{1}{\Gamma(\alpha)} \int_{0}^{\eta} \frac{(\eta-s)^{\alpha}}{\alpha} y(s) \mathrm{d} s+\frac{C_{1} \eta^{\alpha}}{\alpha}
\end{aligned}
$$

yields

$$
C_{1}=\int_{0}^{1} \frac{(1-s)^{\alpha-1}}{\Gamma(\alpha)\left(1-\left(\lambda \eta^{\alpha} / \alpha\right)\right)} y(s) \mathrm{d} s-\lambda \int_{0}^{\eta} \frac{(\eta-s)^{\alpha}}{\alpha \Gamma(\alpha)\left(1-\left(\lambda \eta^{\alpha} / \alpha\right)\right)} y(s) \mathrm{d} s .
$$

Therefore, the unique solution of the problem (2.14) is

$$
\begin{aligned}
u(t)= & -\int_{0}^{t} \frac{(t-s)^{\alpha-1}}{\Gamma(\alpha)} y(s) \mathrm{d} s+\frac{1}{\left(1-\left(\lambda \eta^{\alpha} / \alpha\right)\right)} \int_{0}^{1} \frac{(1-s)^{\alpha-1} t^{\alpha-1}}{\Gamma(\alpha)} y(s) \mathrm{d} s \\
& -\frac{1}{\left(1-\left(\lambda \eta^{\alpha} / \alpha\right)\right)} \int_{0}^{\eta} \frac{(\lambda / \alpha)(\eta-s)^{\alpha} t^{\alpha-1}}{\Gamma(\alpha)} y(s) \mathrm{d} s .
\end{aligned}
$$

For $t \leq \eta$, one has

$$
\begin{aligned}
u(t)= & -\int_{0}^{t} \frac{(t-s)^{\alpha-1}}{\Gamma(\alpha)} y(s) \mathrm{d} s+\frac{1}{\left(1-(\lambda / \alpha) \eta^{\alpha}\right)}\left[\left(\int_{0}^{t}+\int_{t}^{\eta}+\int_{\eta}^{1}\right) \frac{(1-s)^{\alpha-1} t^{\alpha-1}}{\Gamma(\alpha)} y(s) \mathrm{d} s\right] \\
& -\frac{\lambda}{\left(1-(\lambda / \alpha) \eta^{\alpha}\right)}\left[\left(\int_{0}^{t}+\int_{t}^{\eta}\right) \frac{(1 / \alpha)(\eta-s)^{\alpha} t^{\alpha-1}}{\Gamma(\alpha)} y(s) \mathrm{d} s\right] \\
= & \int_{0}^{t} \frac{t^{\alpha-1}(1-s)^{\alpha-1}-(\lambda / \alpha)(\eta-s)^{\alpha} t^{\alpha-1}-\left(1-(\lambda / \alpha) \eta^{\alpha}\right)(t-s)^{\alpha-1}}{\left(1-(\lambda / \alpha) \eta^{\alpha}\right) \Gamma(\alpha)} y(s) \mathrm{d} s \\
& +\int_{t}^{\eta} \frac{t^{\alpha-1}(1-s)^{\alpha-1}-(\lambda / \alpha)(\eta-s)^{\alpha} t^{\alpha-1}}{\left(1-(\lambda / \alpha) \eta^{\alpha}\right) \Gamma(\alpha)} y(s) \mathrm{d} s+\int_{\eta}^{1} \frac{t^{\alpha-1}(1-s)^{\alpha-1}}{\left(1-(\lambda / \alpha) \eta^{\alpha}\right) \Gamma(\alpha)} y(s) \mathrm{d} s \\
= & \int_{0}^{1} G(t, s) y(s) \mathrm{d} s .
\end{aligned}
$$


For $t \geq \eta$, one has

$$
\begin{aligned}
u(t)= & -\left(\int_{0}^{\eta}+\int_{\eta}^{t}\right) \frac{(t-s)^{\alpha-1}}{\Gamma(\alpha)} y(s) \mathrm{d} s+\frac{1}{\left(1-(\lambda / \alpha) \eta^{\alpha}\right)}\left[\left(\int_{0}^{\eta}+\int_{\eta}^{t}+\int_{t}^{1}\right) \frac{(1-s)^{\alpha-1} t^{\alpha-1}}{\Gamma(\alpha)} y(s) \mathrm{d} s\right] \\
& -\frac{\lambda}{\left(1-(\lambda / \alpha) \eta^{\alpha}\right)} \int_{0}^{\eta} \frac{(1 / \alpha)(\eta-s)^{\alpha} t^{\alpha-1}}{\Gamma(\alpha)} y(s) \mathrm{d} s \\
= & \int_{0}^{\eta} \frac{t^{\alpha-1}(1-s)^{\alpha-1}-(\lambda / \alpha)(\eta-s)^{\alpha} t^{\alpha-1}-\left(1-(\lambda / \alpha) \eta^{\alpha}\right)(t-s)^{\alpha-1}}{\left(1-(\lambda / \alpha) \eta^{\alpha}\right) \Gamma(\alpha)} y(s) \mathrm{d} s \\
& +\int_{\eta}^{t} \frac{t^{\alpha-1}(1-s)^{\alpha-1}-\left(1-(\lambda / \alpha) \eta^{\alpha}\right)(t-s)^{\alpha-1}}{\left(1-(\lambda / \alpha) \eta^{\alpha}\right) \Gamma(\alpha)} y(s) \mathrm{d} s+\int_{t}^{1} \frac{t^{\alpha-1}(1-s)^{\alpha-1}}{\left(1-(\lambda / \alpha) \eta^{\alpha}\right) \Gamma(\alpha)} y(s) \mathrm{d} s \\
= & \int_{0}^{1} G(t, s) y(s) \mathrm{d} s .
\end{aligned}
$$

The proof is complete.

Lemma 2.12. The function $G(t, s)$ defined by (2.16) satisfies

(a1) $G(t, s) \geq m_{1} t^{\alpha-1} s(1-s)^{\alpha-1}$, for all $t, s \in[0,1]$;

(a2) $G(t, s) \leq M_{1} t^{\alpha-1}(1-s)^{\alpha-1}$, for all $t, s \in[0,1]$;

(a3) $G(t, s) \leq M_{1} s(1-s)^{\alpha-1}$, for all $t, s \in[0,1]$;

(a4) $p(s)>0$, and $p(s)$ is not decreasing on $[0,1]$;

(a5) $G(t, s)>0$, for all $t, s \in(0,1)$,

$$
\text { where } m_{1}=(1-p(0)) / \Gamma(\alpha) p(0), M_{1}=(\alpha-1) / \Gamma(\alpha)+4(\lambda / \alpha) \eta^{\alpha-1} / p(0) \Gamma(\alpha) \text {. }
$$

Proof. For $s \leq t, s \leq \eta$,

$$
\begin{aligned}
G(t, s) & =\frac{1}{p(0) \Gamma(\alpha)}\left\{[t(1-s)]^{\alpha-1}-\frac{\lambda}{\alpha}(\eta-s)^{\alpha} t^{\alpha-1}-p(0)(t-s)^{\alpha-1}\right\} \\
& =\frac{1}{p(0) \Gamma(\alpha)}\left\{[t(1-s)]^{\alpha-1}-\frac{\lambda}{\alpha} \eta^{\alpha}\left(1-\frac{s}{\eta}\right)^{\alpha} t^{\alpha-1}-p(0)(t-s)^{\alpha-1}\right\} \\
& \geq \frac{1}{p(0) \Gamma(\alpha)}\left\{[t(1-s)]^{\alpha-1}-\frac{\lambda}{\alpha} \eta^{\alpha}(1-s)^{\alpha} t^{\alpha-1}-p(0)(t-s)^{\alpha-1}\right\} \\
& =\frac{1}{p(0) \Gamma(\alpha)}\left\{[t(1-s)]^{\alpha-1}\left[1-\frac{\lambda}{\alpha} \eta^{\alpha}(1-s)\right]-p(0)(t-s)^{\alpha-1}\right\}
\end{aligned}
$$




$$
\begin{aligned}
& =\frac{1}{p(0) \Gamma(\alpha)}\left\{[t(1-s)]^{\alpha-1} p(s)-p(0)(t-s)^{\alpha-1}\right\} \\
& =\frac{1}{\Gamma(\alpha)}\left\{[t(1-s)]^{\alpha-1}-(t-s)^{\alpha-1}\right\}+\frac{p(s)-p(0)}{p(0) \Gamma(\alpha)}[t(1-s)]^{\alpha-1} \\
& =\frac{1}{\Gamma(\alpha)}\left\{[t(1-s)]^{\alpha-2} t(1-s)-(t-s)^{\alpha-2}(t-s)\right\}+\frac{p(s)-p(0)}{p(0) \Gamma(\alpha)}[t(1-s)]^{\alpha-1} \\
& \geq \frac{1}{\Gamma(\alpha)}\left\{[t(1-s)]^{\alpha-2}[t(1-s)-(t-s)]\right\}+\frac{p(s)-p(0)}{p(0) \Gamma(\alpha)}[t(1-s)]^{\alpha-1} \\
& \geq \frac{1}{\Gamma(\alpha)}[t(1-s)]^{\alpha-1} s(1-t)+\frac{1-p(0)}{p(0) \Gamma(\alpha)} s[t(1-s)]^{\alpha-1} \\
& \geq \frac{1-p(0)}{p(0) \Gamma(\alpha)} t^{\alpha-1} s(1-s)^{\alpha-1}, \\
& G(t, s)=\frac{1}{p(0) \Gamma(\alpha)}\left\{[t(1-s)]^{\alpha-1}-\frac{\lambda}{\alpha}(\eta-s)^{\alpha} t^{\alpha-1}-p(0)(t-s)^{\alpha-1}\right\} \\
& =\frac{1}{p(0) \Gamma(\alpha)}\left\{\left(1-\frac{\lambda}{\alpha} \eta^{\alpha}+\frac{\lambda}{\alpha} \eta^{\alpha}\right)[t(1-s)]^{\alpha-1}-\frac{\lambda}{\alpha}(\eta-s)^{\alpha} t^{\alpha-1}-p(0)(t-s)^{\alpha-1}\right\} \\
& =\frac{1}{p(0) \Gamma(\alpha)}\left\{p(0)\left[t^{\alpha-1}(1-s)^{\alpha-1}-(t-s)^{\alpha-1}\right]\right. \\
& \left.+\frac{\lambda}{\alpha} \eta^{\alpha}\left[t^{\alpha-1}(1-s)^{\alpha-1}-\left(1-\frac{s}{\eta}\right)^{\alpha} t^{\alpha-1}\right]\right\} \\
& =\frac{1}{p(0) \Gamma(\alpha)}\left\{p(0)(\alpha-1) \int_{t-s}^{t(1-s)} x^{\alpha-2} \mathrm{~d} x\right. \\
& \left.+\frac{\lambda}{\alpha} \eta^{\alpha}\left[t^{\alpha-1}(1-s)^{\alpha-1}-\left(1-\frac{s}{\eta}\right)^{\alpha} t^{\alpha-1}(1-s)^{\alpha-1}\right]\right\} \\
& \leq \frac{1}{p(0) \Gamma(\alpha)}\left\{p(0)(\alpha-1) t^{\alpha-2}(1-s)^{\alpha-2} s(1-t)+\frac{\lambda}{\alpha} \eta^{\alpha} t^{\alpha-1}(1-s)^{\alpha-1}\left[1-\left(1-\frac{s}{\eta}\right)^{\alpha}\right]\right\} \\
& \leq \frac{1}{p(0) \Gamma(\alpha)}\left\{p(0)(\alpha-1) t^{\alpha-2}(1-s)^{\alpha-2} s(1-t)+\frac{\lambda}{\alpha} \eta^{\alpha} t^{\alpha-1}(1-s)^{\alpha-1}\left[1-\left(1-\frac{s}{\eta}\right)^{4}\right]\right\} \\
& \leq \frac{1}{p(0) \Gamma(\alpha)}\left\{p(0)(\alpha-1) t^{\alpha-1}(1-s)^{\alpha-1}+\frac{\lambda}{\alpha} \eta^{\alpha} t^{\alpha-1}(1-s)^{\alpha-1}\right. \\
& \left.\times\left[1-\left(1-\frac{s}{\eta}\right)\right]\left[1+\left(1-\frac{s}{\eta}\right)\right]\left[1+\left(1-\frac{s}{\eta}\right)^{2}\right]\right\} \\
& \leq \frac{1}{p(0) \Gamma(\alpha)}\left\{p(0)(\alpha-1) t^{\alpha-1}(1-s)^{\alpha-1}+4 \frac{\lambda}{\alpha} \eta^{\alpha-1} t^{\alpha-1}(1-s)^{\alpha-1}\right\} \\
& =\left[\frac{\alpha-1}{\Gamma(\alpha)}+\frac{4(\lambda / \alpha) \eta^{\alpha-1}}{p(0) \Gamma(\alpha)}\right] t^{\alpha-1}(1-s)^{\alpha-1}
\end{aligned}
$$




$$
\begin{aligned}
G(t, s) & =\frac{1}{p(0) \Gamma(\alpha)}\left\{[t(1-s)]^{\alpha-1}-\frac{\lambda}{\alpha}(\eta-s)^{\alpha} t^{\alpha-1}-p(0)(t-s)^{\alpha-1}\right\} \\
& =\frac{1}{p(0) \Gamma(\alpha)}\left\{\left(1-\frac{\lambda}{\alpha} \eta^{\alpha}+\frac{\lambda}{\alpha} \eta^{\alpha}\right)[t(1-s)]^{\alpha-1}-\frac{\lambda}{\alpha}(\eta-s)^{\alpha} t^{\alpha-1}-p(0)(t-s)^{\alpha-1}\right\} \\
& =\frac{1}{p(0) \Gamma(\alpha)}\left\{p(0)\left[t^{\alpha-1}(1-s)^{\alpha-1}-(t-s)^{\alpha-1}\right]+\frac{\lambda}{\alpha} \eta^{\alpha}\left[t^{\alpha-1}(1-s)^{\alpha-1}-\left(1-\frac{s}{\eta}\right)^{\alpha} t^{\alpha-1}\right]\right\} \\
& \leq \frac{1}{p(0) \Gamma(\alpha)}\left\{p(0)(\alpha-1) \int_{t-s}^{t(1-s)} x^{\alpha-2} \mathrm{~d} x+\frac{\lambda}{\alpha} \eta^{\alpha}\left[t^{\alpha-1}(1-s)^{\alpha-1}-\left(1-\frac{s}{\eta}\right)^{\alpha} t^{\alpha-1}(1-s)^{\alpha-1}\right]\right\} \\
& \leq \frac{1}{p(0) \Gamma(\alpha)}\left\{p(0)(\alpha-1) t^{\alpha-2}(1-s)^{\alpha-2} s(1-t)+\frac{\lambda}{\alpha} \eta^{\alpha} t^{\alpha-1}(1-s)^{\alpha-1}\left[1-\left(1-\frac{s}{\eta}\right)^{\alpha}\right]\right\} \\
& \leq \frac{1}{p(0) \Gamma(\alpha)}\left\{p(0)(\alpha-1) t^{\alpha-2}(1-s)^{\alpha-2} s(1-t)+\frac{\lambda}{\alpha} \eta^{\alpha} t^{\alpha-1}(1-s)^{\alpha-1}\left[1-\left(1-\frac{s}{\eta}\right)^{4}\right]\right\} \\
& \leq \frac{1}{p(0) \Gamma(\alpha)}\left\{p(0)(\alpha-1) t^{\alpha-2}(1-s)^{\alpha-2} s(1-t)+\frac{\lambda}{\alpha} \eta^{\alpha} t^{\alpha-1}(1-s)^{\alpha-1}\right. \\
& \left.\quad \times\left[1-\left(1-\frac{s}{\eta}\right)\right]\left[1+\left(1-\frac{s}{\eta}\right)\right]\left[1+\left(1-\frac{s}{\eta}\right)^{2}\right]\right\} \\
& \leq \frac{1}{p(0) \Gamma(\alpha)}\left\{p(0)(\alpha-1)(1-s)^{\alpha-1} s+4 \frac{\lambda}{\alpha} \eta^{\alpha-1} s(1-s)^{\alpha-1}\right\} \\
& {\left[\frac{\alpha-1}{\Gamma(\alpha)}+\frac{4(\lambda / \alpha) \eta^{\alpha-1}}{p(0) \Gamma(\alpha)}\right] s(1-s)^{\alpha-1} . }
\end{aligned}
$$

For $\eta \leq s \leq t$,

$$
\begin{aligned}
G(t, s) & =\frac{1}{p(0) \Gamma(\alpha)}\left\{[t(1-s)]^{\alpha-1}-p(0)(t-s)^{\alpha-1}\right\} \\
& \geq \frac{p(s)}{p(0) \Gamma(\alpha)}[t(1-s)]^{\alpha-1}-\frac{1}{\Gamma(\alpha)}(t-s)^{\alpha-1} \\
& =\frac{1}{\Gamma(\alpha)}\left\{[t(1-s)]^{\alpha-2} t(1-s)-(t-s)^{\alpha-2}(t-s)\right\}+\frac{p(s)-p(0)}{p(0) \Gamma(\alpha)}[t(1-s)]^{\alpha-1} \\
& \geq \frac{1}{\Gamma(\alpha)}[t(1-s)]^{\alpha-1} s(1-t)+\frac{1-p(0)}{p(0) \Gamma(\alpha)} s[t(1-s)]^{\alpha-1} \\
& \geq \frac{1-p(0)}{p(0) \Gamma(\alpha)} t^{\alpha-1} s(1-s)^{\alpha-1}
\end{aligned}
$$




$$
\begin{aligned}
& G(t, s)=\frac{1}{p(0) \Gamma(\alpha)}\left\{[t(1-s)]^{\alpha-1}-p(0)(t-s)^{\alpha-1}\right\} \\
& =\frac{1}{p(0) \Gamma(\alpha)}\left\{\left(1-\frac{\lambda}{\alpha} \eta^{\alpha}+\frac{\lambda}{\alpha} \eta^{\alpha}\right)[t(1-s)]^{\alpha-1}-p(0)(t-s)^{\alpha-1}\right\} \\
& =\frac{1}{p(0) \Gamma(\alpha)}\left\{p(0)\left[t^{\alpha-1}(1-s)^{\alpha-1}-(t-s)^{\alpha-1}\right]+\frac{\lambda}{\alpha} \eta^{\alpha}[t(1-s)]^{\alpha-1}\right\} \\
& \leq \frac{1}{p(0) \Gamma(\alpha)}\left\{p(0)(\alpha-1) \int_{t-s}^{t(1-s)} x^{\alpha-2} \mathrm{~d} x+\frac{\lambda}{\alpha} \eta^{\alpha-1} s t^{\alpha-1}(1-s)^{\alpha-1}\right\} \\
& \leq \frac{1}{p(0) \Gamma(\alpha)}\left\{p(0)(\alpha-1) t^{\alpha-2}(1-s)^{\alpha-2} s(1-t)+\frac{\lambda}{\alpha} \eta^{\alpha-1} s t^{\alpha-1}(1-s)^{\alpha-1}\right\} \\
& \leq \frac{1}{p(0) \Gamma(\alpha)}\left\{p(0)(\alpha-1) t^{\alpha-1}(1-s)^{\alpha-1}+\frac{\lambda}{\alpha} \eta^{\alpha-1} t^{\alpha-1}(1-s)^{\alpha-1}\right\} \\
& \leq\left[\frac{\alpha-1}{\Gamma(\alpha)}+\frac{4(\lambda / \alpha) \eta^{\alpha-1}}{p(0) \Gamma(\alpha)}\right] t^{\alpha-1}(1-s)^{\alpha-1} \\
& G(t, s)=\frac{1}{p(0) \Gamma(\alpha)}\left\{[t(1-s)]^{\alpha-1}-p(0)(t-s)^{\alpha-1}\right\} \\
& =\frac{1}{p(0) \Gamma(\alpha)}\left\{\left(1-\frac{\lambda}{\alpha} \eta^{\alpha}+\frac{\lambda}{\alpha} \eta^{\alpha}\right)[t(1-s)]^{\alpha-1}-p(0)(t-s)^{\alpha-1}\right\} \\
& =\frac{1}{p(0) \Gamma(\alpha)}\left\{p(0)\left[t^{\alpha-1}(1-s)^{\alpha-1}-(t-s)^{\alpha-1}\right]+\frac{\lambda}{\alpha} \eta^{\alpha}[t(1-s)]^{\alpha-1}\right\} \\
& \leq \frac{1}{p(0) \Gamma(\alpha)}\left\{p(0)(\alpha-1) \int_{t-s}^{t(1-s)} x^{\alpha-2} \mathrm{~d} x+\frac{\lambda}{\alpha} \eta^{\alpha-1} s t^{\alpha-1}(1-s)^{\alpha-1}\right\} \\
& \leq \frac{1}{p(0) \Gamma(\alpha)}\left\{p(0)(\alpha-1) t^{\alpha-2}(1-s)^{\alpha-2} s(1-t)+\frac{\lambda}{\alpha} \eta^{\alpha-1} s t^{\alpha-1}(1-s)^{\alpha-1}\right\} \\
& \leq \frac{1}{p(0) \Gamma(\alpha)}\left\{p(0)(\alpha-1)(1-s)^{\alpha-1} s+\frac{\lambda}{\alpha} \eta^{\alpha-1} s(1-s)^{\alpha-1}\right\} \\
& \leq\left[\frac{\alpha-1}{\Gamma(\alpha)}+\frac{4(\lambda / \alpha) \eta^{\alpha-1}}{p(0) \Gamma(\alpha)}\right] s(1-s)^{\alpha-1} \text {. }
\end{aligned}
$$

For $t \leq s \leq \eta$,

$$
\begin{aligned}
G(t, s) & =\frac{1}{p(0) \Gamma(\alpha)}\left\{[t(1-s)]^{\alpha-1}-\frac{\lambda}{\alpha}(\eta-s)^{\alpha} t^{\alpha-1}\right\} \\
& =\frac{1}{p(0) \Gamma(\alpha)}\left\{[t(1-s)]^{\alpha-1}-\frac{\lambda}{\alpha} \eta^{\alpha}\left(1-\frac{s}{\eta}\right)^{\alpha} t^{\alpha-1}\right\}
\end{aligned}
$$


Abstract and Applied Analysis

$$
\begin{aligned}
& \geq \frac{1}{p(0) \Gamma(\alpha)}\left\{[t(1-s)]^{\alpha-1}-\frac{\lambda}{\alpha} \eta^{\alpha}(1-s)^{\alpha} t^{\alpha-1}\right\} \\
& =\frac{1}{p(0) \Gamma(\alpha)}[t(1-s)]^{\alpha-1}\left[1-\frac{\lambda}{\alpha} \eta^{\alpha}(1-s)\right] \\
& =\frac{p(0)+p(s)-p(0)}{p(0) \Gamma(\alpha)}[t(1-s)]^{\alpha-1} \\
& =\frac{1}{\Gamma(\alpha)}[t(1-s)]^{\alpha-1}+\frac{p(s)-p(0)}{p(0) \Gamma(\alpha)}[t(1-s)]^{\alpha-1} \\
& \geq \frac{1-p(0)}{p(0) \Gamma(\alpha)} t^{\alpha-1} s(1-s)^{\alpha-1}, \\
G(t, s) & =\frac{1}{p(0) \Gamma(\alpha)}\left\{[t(1-s)]^{\alpha-1}-\frac{\lambda}{\alpha}(\eta-s)^{\alpha} t^{\alpha-1}\right\} \\
& \leq \frac{1}{p(0) \Gamma(\alpha)}[t(1-s)]^{\alpha-1} \\
& \leq\left[\frac{\alpha-1}{\Gamma(\alpha)}+\frac{4(\lambda / \alpha) \eta^{\alpha-1}}{p(0) \Gamma(\alpha)}\right] t^{\alpha-1}(1-s)^{\alpha-1}, \\
& \leq\left[\frac{\alpha-1}{\Gamma(\alpha)}+\frac{4(\lambda / \alpha) \eta^{\alpha-1}}{p(0) \Gamma(\alpha)}\right] s(1-s)^{\alpha-1} . \\
G(t, s)= & \frac{1}{p(0) \Gamma(\alpha)}\left\{[t(1-s)]^{\alpha-1}-\frac{\lambda}{\alpha}(\eta-s)^{\alpha} t^{\alpha-1}\right\} \\
& {[t(1-s)]^{\alpha-1} }
\end{aligned}
$$

For $\eta \leq s, t \leq s$,

$$
\begin{aligned}
G(t, s) & =\frac{1}{p(0) \Gamma(\alpha)}[t(1-s)]^{\alpha-1} \\
& \geq \frac{1-p(0)}{p(0) \Gamma(\alpha)} t^{\alpha-1} s(1-s)^{\alpha-1}, \\
G(t, s) & =\frac{1}{p(0) \Gamma(\alpha)}[t(1-s)]^{\alpha-1} \\
& \leq\left[\frac{\alpha-1}{\Gamma(\alpha)}+\frac{4(\lambda / \alpha) \eta^{\alpha-1}}{p(0) \Gamma(\alpha)}\right] t^{\alpha-1}(1-s)^{\alpha-1},
\end{aligned}
$$




$$
\begin{aligned}
G(t, s) & =\frac{1}{p(0) \Gamma(\alpha)}[t(1-s)]^{\alpha-1} \\
& \leq\left[\frac{\alpha-1}{\Gamma(\alpha)}+\frac{4(\lambda / \alpha) \eta^{\alpha-1}}{p(0) \Gamma(\alpha)}\right] s(1-s)^{\alpha-1} .
\end{aligned}
$$

From above, (a1), (a2), (a3), (a5) are complete. Clearly, (a4) is true. The proof is complete.

\section{Main Results and Proof}

Let $E=C[0,1],\|u\|=\max _{t \in[0,1]}|u(t)|, P=\{u \in C[0,1] \mid u(t) \geq 0, t \in[0,1]\}$. Obviously, $P$ is a normal solid cone with normal constant 1 in Banach space $E$, and $E$ is a lattice under the partial ordering $\leq$ which is deduced by $P$.

Throughout this paper, we always assume that

(H1) $f:[0,1] \times R \rightarrow R$ is continuous;

$(\mathrm{H} 2) h:(0,1) \rightarrow[0,+\infty)$ is continuous and not identical zero on any closed subinterval of $[0,+\infty)$ with $0<\int_{0}^{1} h(t) t(1-t)^{\alpha-1} \mathrm{~d} t<+\infty$.

Remark 3.1. In the assumption (H1), it is not required that $f(t, u) \geq 0, \forall u \geq 0$.

Define operators $A$ and $B$ as follows:

$$
\begin{gathered}
(A u)(t)=\int_{0}^{1} G(t, s) h(s) f(s, u(s)) \mathrm{d} s, \quad(B u)(t)=\int_{0}^{1} G(t, s) h(s) u(s) \mathrm{d} s, \quad t \in[0,1] \\
(F u)(t)=f(t, u(t)) .
\end{gathered}
$$

Remark 3.2. By Lemma 2.12, (H1) and (H2), it is easy to see that operators $A$ and $B$ defined by (3.1) are well defined.

Lemma 3.3. Suppose that $(H 2)$ holds, then the spectral radius $r(B) \neq 0$ and $B$ has a positive eigenfunction corresponding to the first eigenvalue $\lambda_{1}=(r(B))^{-1}$.

Proof. By Lemma 2.11, (H2), similar to the proof of Lemma 4.4 in [28], the proof can be easily given. We omit the details.

By standard argument, we have the following.

Lemma 3.4. Suppose that (H1) and (H2) hold, then $A: E \rightarrow E$ is completely continuous. 
Theorem 3.5. Suppose that conditions (H1) and (H2) are satisfied. If there exists a constant b such that

$$
\begin{gathered}
f(t, u) \geq-b, \quad \forall t \in[0,1], u \geq 0 \\
\lim _{|u| \rightarrow+\infty} \sup \frac{f(t, u)}{u}<\lambda_{1} \quad \text { uniformly for } t \in[0,1]
\end{gathered}
$$

where $\lambda_{1}$ is the first eigenvalue of B defined by (3.1), then BVP (1.1) has at least one solution.

Proof. From Lemma 3.4, we know that $A: E \rightarrow E$ is completely continuous. By (3.4), there exist $R_{0}>0, \lambda_{1}>\varepsilon>0$ such that

$$
\begin{aligned}
& f(t, u) \leq\left(\lambda_{1}-\varepsilon\right) u, \quad t \in[0,1], u \geq R_{0} \\
& f(t, u) \geq\left(\lambda_{1}-\varepsilon\right) u, \quad t \in[0,1], u \leq-R_{0} .
\end{aligned}
$$

This implies

$$
\begin{aligned}
& f(t, u) \leq\left(\lambda_{1}-\varepsilon\right) u+M_{2}, \quad t \in[0,1], u \geq 0, \\
& f(t, u) \geq\left(\lambda_{1}-\varepsilon\right) u-M_{2}, \quad t \in[0,1], u \leq 0,
\end{aligned}
$$

where $M_{2}=\max _{0 \leq t \leq 1,|u| \leq R_{0}}|f(t, u)|$. Set

$$
u^{*}(t)=b \int_{0}^{1} G(t, s) h(s) \mathrm{d} s, \quad u_{1}(t)=M_{2} \int_{0}^{1} G(t, s) h(s) \mathrm{d} s
$$

Obviously, $u^{*}, u_{1} \in P$. Let $B_{0}=\left(\lambda_{1}-\varepsilon\right) B$, where $B$ is defined as (3.1). It is clear that $B_{0}: P \rightarrow P$ is a positive bounded linear operator and

$$
r\left(B_{0}\right)=r\left(\left(\lambda_{1}-\varepsilon\right) B\right)<\lambda_{1} r(B)=1
$$

It follows from (3.3), (3.6) that

$$
\begin{gathered}
-u^{*} \leq A u \leq B_{0} u+u_{1}, \quad \forall u \in P, \\
A u \geq B_{0} u-u_{1}, \quad \forall u \in-P .
\end{gathered}
$$

It follows from Lemma 2.5 that there exists $R>0$ big enough such that

$$
\operatorname{deg}\left(I-A, B_{R}, \theta\right)=1
$$

which means that $A$ has at least one solution. 
Theorem 3.6. Suppose that (H1) and (H2) hold. In addition,

$$
\lim _{|u| \rightarrow+\infty} \sup \frac{|f(t, u)|}{|u|}<\lambda_{1} \quad \text { uniformly for } t \in[0,1]
$$

Then BVP (1.1) has at least one solution.

Proof. Similar to the proof of (3.9), we arrive at

$$
|A u| \leq B|u|+u_{1}, \quad \forall u \in C[0,1] .
$$

By Lemma 2.6, there exists $R>0$ big enough such that

$$
\operatorname{deg}\left(I-A, B_{R}, \theta\right)=1,
$$

which shows that $A$ has at least one solution.

Theorem 3.7. Suppose that conditions (H1) and (H2) are satisfied. If

$$
\begin{gathered}
\liminf _{u \rightarrow+\infty} \frac{f(t, u)}{u}>\lambda_{1}, \quad \text { uniformly on } t \in[0,1] ; \\
\limsup _{u \rightarrow-\infty} \frac{f(t, u)}{u}<\lambda_{1}, \quad \text { uniformly on } t \in[0,1] \\
\limsup _{u \rightarrow 0}\left|\frac{f(t, u)}{u}\right|<\lambda_{1}, \quad \text { uniformly on } t \in[0,1],
\end{gathered}
$$

where $\lambda_{1}$ is the first eigenvalue of B defined by (3.1), then the singular BVP (1.1) has at least one nontrivial solution.

Proof. Let $E=C[0,1]$, and let $A, B$ and $F$ be defined by (3.1) and (3.2), respectively. Obviously, by remark 3.1 in $[27,28], F: E \rightarrow E$ is continuous and quasiadditive on lattice, and $A=B F$. By Lemma 3.4, we know that $A: E \rightarrow E$ is completely continuous.

It follows from (3.14) and (3.15) that there exist constants $\varepsilon>0$ and $R_{0}>$ such that

$$
\begin{gathered}
f(t, u) \geq\left(\lambda_{1}+\varepsilon\right) u, \quad t \in[0,1], u \geq R_{0} \\
f(t, u) \geq\left(\lambda_{1}-\varepsilon\right) u, \quad t \in[0,1], u \leq-R_{0} .
\end{gathered}
$$

Therefore, there exists a constant $M_{3}>0$ such that

$$
\begin{aligned}
& f(t, u) \geq\left(\lambda_{1}+\varepsilon\right) u-M_{3}, \quad t \in[0,1], u \geq 0 \\
& f(t, u) \geq\left(\lambda_{1}-\varepsilon\right) u-M_{3}, \quad t \in[0,1], u \leq 0 .
\end{aligned}
$$

From (3.18), one can see that (2.9) and (2.10) hold for $a_{1}=\lambda_{1}+\varepsilon>\lambda_{1}=r^{-1}(B)$ and $a_{2}=$ $\lambda_{1}-\varepsilon<\lambda_{1}=r^{-1}(B)$. 
Next, we are in position to show that $B$ satisfies $H$ condition. Let

$$
\left(B^{*} u\right)(t)=\int_{0}^{1} G(s, t) h(t) u(s) \mathrm{d} s
$$

By Lemma 3.3, we know that $r\left(B^{*}\right)=r(B) \neq 0$, and there exits $g^{*} \in P^{*} \backslash\{\theta\}$, such that

$$
g^{*}=r^{-1}(B) B^{*} g^{*}
$$

By Lemma 2.12, we have

$$
\begin{aligned}
g^{*}(s) & =r^{-1}(B) B^{*} g^{*} \\
& =r^{-1}(B) \int_{0}^{1} G(t, s) h(s) g^{*}(t) \mathrm{d} t \\
& \geq m_{1} r^{-1}(B) \int_{0}^{1} t^{\alpha-1} s(1-s)^{\alpha-1} g^{*}(t) \mathrm{d} t \\
& =\left[m_{1} r^{-1}(B) \int_{0}^{1} t^{\alpha-1} g^{*}(t) \mathrm{d} t\right] s(1-s)^{\alpha-1}, \quad \forall s \in[0,1] .
\end{aligned}
$$

Therefore, for $u \in P$, we get by Lemma 2.12, (3.20) and (3.21) that

$$
\begin{aligned}
\int_{0}^{1} g^{*}(t)(B u)(t) \mathrm{d} t & =\iint_{0}^{1} g^{*}(t) G(t, s) h(s) u(s) \mathrm{d} s \mathrm{~d} t \\
& =\int_{0}^{1}\left[\int_{0}^{1} g^{*}(t) G(t, s) h(s) \mathrm{d} t\right] u(s) \mathrm{d} s \\
& =r(B) \int_{0}^{1} g^{*}(s) u(s) \mathrm{d} s \\
& \geq r(B) m_{1} r^{-1}(B) \int_{0}^{1} t^{\alpha-1} g^{*}(t) \mathrm{d} t \cdot \int_{0}^{1} s(1-s)^{\alpha-1} u(s) \mathrm{d} s \\
& \geq \frac{m_{1}}{M_{1}} \int_{0}^{1} t^{\alpha-1} g^{*}(t) \mathrm{d} t \cdot M_{1} \int_{0}^{1} s(1-s)^{\alpha-1} u(s) \mathrm{d} s \\
& \geq \frac{m_{1}}{M_{1}} \int_{0}^{1} t^{\alpha-1} g^{*}(t) \mathrm{d} t \cdot\|B u\| .
\end{aligned}
$$

This means that $g^{*}(B u) \geq \delta\|B u\|$, where $\delta=\left(m_{1} / M_{1}\right) \int_{0}^{1} t^{\alpha-1} g^{*}(t) \mathrm{d} t$. So, $B(P) \subset P\left(g^{*}, \delta\right)$. Thus, we have shown that $B$ satisfies $H$ condition. By Lemma 2.7, we know that there exists $R>R_{0}$ such that

$$
\operatorname{deg}\left(I-A, T_{R}, \theta\right)=0
$$


On the other hand, by (3.16), we know that there exists $0<r<R_{0}$ such that

$$
|f(t, u)| \leq\left(\lambda_{1}-\varepsilon\right)|u|, \quad \forall t \in[0,1],|u| \leq r
$$

which implies that

$$
|A u| \leq B_{0}|u|, \quad \forall u \in \partial T_{r}
$$

where $B_{0}=\left(\lambda_{1}-\varepsilon\right) B$, and $r\left(B_{0}\right)=\left(\lambda_{1}-\varepsilon\right) r(B)<1$. By virtue of Lemma 2.8, we get that

$$
\operatorname{deg}\left(I-A, T_{r}, \theta\right)=1
$$

It follows from the additivity of the topology degree and Lemma 2.11 that $A$ has at least one nontrivial fixed point in $\bar{T}_{R} \backslash T_{r}$. That is, BVP (1.1) has at least one nontrivial solution.

\section{An Example}

Consider the following fractional differential equations with integral boundary conditions:

$$
\begin{gathered}
D_{0+}^{7 / 2} u(t)+\lambda_{0} t^{p-1}(1-t)^{q-1}\left[\left(\sqrt{u^{2}+1}-1\right)-\sin \left(u+\frac{\pi}{2}\right)\right]=0, \quad 0<t<1, \\
u(0)=u^{\prime}(0)=u^{\prime \prime}(0)=0, \\
u(1)=\int_{0}^{1 / 2} u(s) \mathrm{d} s,
\end{gathered}
$$

where $0<p, q<1, \lambda_{0}<\lambda_{1}, \lambda_{1}$ is the first eigenvalue of operator $B$. It is easy to see that (H1) and (H2) hold for $h(t)=t^{p-1}(1-t)^{q-1}, f(t, u)=\lambda_{0}\left(\sqrt{u^{2}+1}-1\right)-\sin (u+\pi / 2)$. By Lemma 3.3, we get that $\lambda_{1}>0$. Obviously, $f(u) \geq-1$ is bounded below and sign-changing for $u \geq 0$. By direct computation, we have $\lim _{u \rightarrow+\infty}(f(u) / u)=\lambda_{0}<\lambda_{1}, \lim _{u \rightarrow-\infty}(f(u) / u)=-\lambda_{0}<\lambda_{1}$. Thus (3.3) and (3.4) in Theorem 3.5 hold. It follows from Theorem 3.5 that BVP (4.1) has one solution.

\section{Acknowledgments}

The authors thank the referee for their valuable comments and suggestions. The project is supported financially by the Foundation for Outstanding Middle-Aged and Young Scientists of Shandong Province (BS2010SF004), the National Natural Science Foundation of China (10971179), and a Project of Shandong Province Higher Educational Science and Technology Program (no. J10LA53).

\section{References}

[1] S. G. Samko, A. A. Kilbas, and O. I. Marichev, Fractional Integrals and Derivatives, Theory and Applications, Gordon and Breach Science, Yverdon, Switzerland, 1993. 
[2] I. Podlubny, Fractional Differential Equations, vol. 198 of Mathematics in Science and Engineering, Academic Press, NewYork, NY, USA, 1999.

[3] A. A. Kilbas, H. M. Srivastava, and J. J. Trujillo, Theory and Applications of Fractional Differential Equations, vol. 204 of North-Holland Mathematics Studies, Elsevier Science, Amsterdam, The Netherlands, 2006.

[4] D. Delbosco and L. Rodino, "Existence and uniqueness for a nonlinear fractional differential equation," Journal of Mathematical Analysis and Applications, vol. 204, no. 2, pp. 609-625, 1996.

[5] S. Zhang, "The existence of a positive solution for a nonlinear fractional differential equation," Journal of Mathematical Analysis and Applications, vol. 252, no. 2, pp. 804-812, 2000.

[6] S. Zhang, "Existence of positive solutions for some class of nonlinear fractional equation," Journal of Mathematical Analysis and Applications, vol. 278, pp. 136-148, 2003.

[7] H. Jafari and V. Daftardar-Gejji, "Positive solutions of nonlinear fractional boundary value problems using Adomian decomposition method," Applied Mathematics and Computation, vol. 180, no. 2, pp. 700-706, 2006.

[8] Z. Wei, Q. Li, and J. Che, "Initial value problems for fractional differential equations involving Riemann-Liouville sequential fractional derivative," Journal of Mathematical Analysis and Applications, vol. 367, no. 1, pp. 260-272, 2010.

[9] S. Liang and J. Zhang, "Positive solutions for boundary value problems of nonlinear fractional differential equation," Nonlinear Analysis. Theory, Methods E Applications, vol. 71, no. 11, pp. 55455550, 2009.

[10] S. Liang and J. Zhang, "Existence of multiple positive solutions for $m$-point fractional boundary value problems on an infinite interval," Mathematical and Computer Modelling, vol. 54, no. 5-6, pp. 1334-1346, 2011.

[11] S. Liang and J. Zhang, "Existence and uniqueness of strictly nondecreasing and positive solution for a fractional three-point boundary value problem," Computers \& Mathematics with Applications, vol. 62, no. 3, pp. 1333-1340, 2011.

[12] S. Zhang, "Positive solutions for boundary-value problems of nonlinear fractional differential equations," Electronic Journal of Differential Equations, vol. 36, pp. 1-12, 2006.

[13] Y. Zhao, S. Sun, Z. Han, and M. Zhang, "Positive solutions for boundary value problems of nonlinear fractional differential equations," Applied Mathematics and Computation, vol. 217, no. 16, pp. 6950-6958, 2011.

[14] X. Xu, D. Jiang, and C. Yuan, "Multiple positive solutions for the boundary value problem of a nonlinear fractional differential equation," Nonlinear Analysis. Theory, Methods \& Applications, vol. 71, no. 10, pp. 4676-4688, 2009.

[15] B. Ahmad and G. Wang, "A study of an impulsive four-point nonlocal boundary value problem of nonlinear fractional differential equations," Computers \& Mathematics with Applications, vol. 62, no. 3, pp. 1341-1349, 2011.

[16] M. Feng, X. Liu, and H. Feng, "The existence of positive solution to a nonlinear fractional differential equation with integral boundary conditions," Advances in Difference Equations, vol. 2011, Article ID 546038, 14 pages, 2011.

[17] G. Zhang and J. Sun, "Positive solutions of $m$-point boundary value problems," Journal of Mathematical Analysis and Applications, vol. 291, no. 2, pp. 406-418, 2004.

[18] J. Sun and G. Zhang, "Nontrivial solutions of singular sublinear Sturm-Liouville problems," Journal of Mathematical Analysis and Applications, vol. 326, no. 1, pp. 242-251, 2007.

[19] X. Hao, L. Liu, Y. Wu, and Q. Sun, "Positive solutions for nonlinear $n$ th-order singular eigenvalue problem with nonlocal conditions," Nonlinear Analysis. Theory, Methods \& Applications, vol. 73, no. 6, pp. 1653-1662, 2010.

[20] B. Liu, L. Liu, and Y. Wu, "Positive solutions for singular second order three-point boundary value problems," Nonlinear Analysis. Theory, Methods \& Applications, vol. 66, no. 12, pp. 2756-2766, 2007.

[21] F. Xu, H. Su, and X. Zhang, "Positive solutions of fourth-order nonlinear singular boundary value problems," Nonlinear Analysis. Theory, Methods E Applications, vol. 68, no. 5, pp. 1284-1297, 2008.

[22] H. Ma, "Symmetric positive solutions for nonlocal boundary value problems of fourth order," Nonlinear Analysis. Theory, Methods E Applications, vol. 68, no. 3, pp. 645-651, 2008.

[23] S. Wang and J. Liu, "Coexistence of positive solutions of nonlinear three-point boundary value and its conjugate problem," Journal of Mathematical Analysis and Applications, vol. 330, no. 1, pp. 334-351, 2007. 
[24] L. Liu, B. Liu, and Y. Wu, "Nontrivial solutions of $m$-point boundary value problems for singular second-order differential equations with a sign-changing nonlinear term," Journal of Computational and Applied Mathematics, vol. 224, no. 1, pp. 373-382, 2009.

[25] J. Sun and G. Zhang, "Nontrivial solutions of singular superlinear Sturm-Liouville problems," Journal of Mathematical Analysis and Applications, vol. 313, no. 2, pp. 518-536, 2006.

[26] G. Han and Y. Wu, "Nontrivial solutions of singular two-point boundary value problems with signchanging nonlinear terms," Journal of Mathematical Analysis and Applications, vol. 325, no. 2, pp. 13271338, 2007.

[27] J. Xu, Z. Wei, and W. Dong, “Uniqueness of positive solutions for a class of fractional boundary value problems," Applied Mathematics Letters, vol. 25, no. 3, pp. 590-593, 2012.

[28] Z. Bai, "On positive solutions of a nonlocal fractional boundary value problem," Nonlinear Analysis. Theory, Methods E Applications, vol. 72, no. 2, pp. 916-924, 2010.

[29] J. Sun and X. Liu, "Computation of topological degree for nonlinear operators and applications," Nonlinear Analysis. Theory, Methods E Applications, vol. 69, no. 11, pp. 4121-4130, 2008.

[30] J. Sun and X. Liu, "Computation of topological degree in ordered Banach spaces with lattice structure and its application to superlinear differential equations," Journal of Mathematical Analysis and Applications, vol. 348, no. 2, pp. 927-937, 2008.

[31] K. Deimling, Nonlinear Functional Analysis, Springer, Berlin, Germany, 1985.

[32] D. J. Guo and V. Lakshmikantham, Nonlinear Problems in Abstract Cones, vol. 5, Academic Press, San Diego, Calif, USA, 1988.

[33] W. A. J. Lucxemburg and A. C. Zaanen, Riesz Space, vol. 1, North-Holland Publishing, London, UK.

[34] M. G. Krein and M. A. Rutman, "Linear operators leaving invariant a cone in a Banach space," American Mathematical Society Translations, vol. 10, no. 26, pp. 199-325, 1962. 


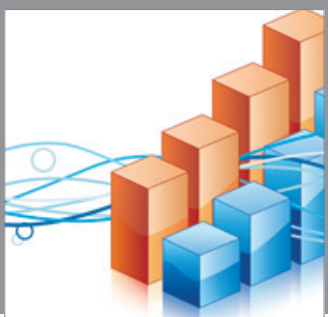

Advances in

Operations Research

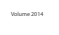

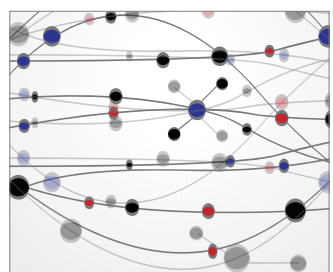

\section{The Scientific} World Journal
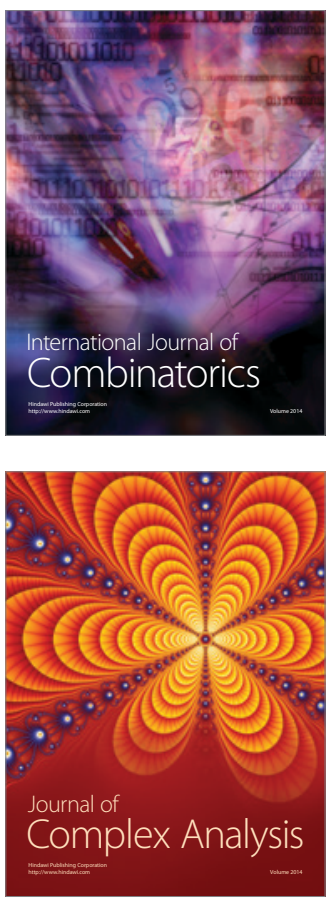

International Journal of

Mathematics and

Mathematical

Sciences
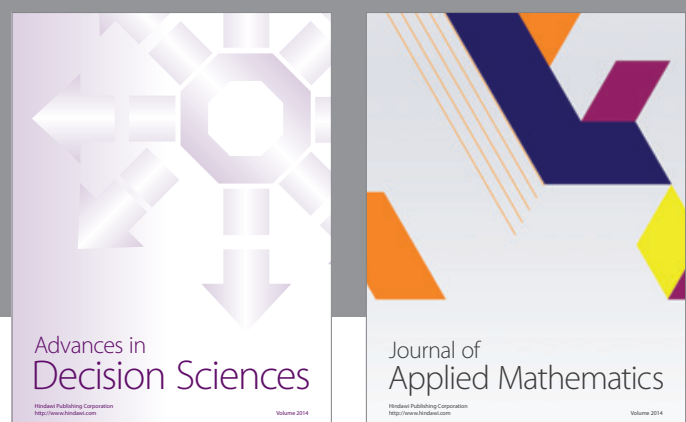

Journal of

Applied Mathematics
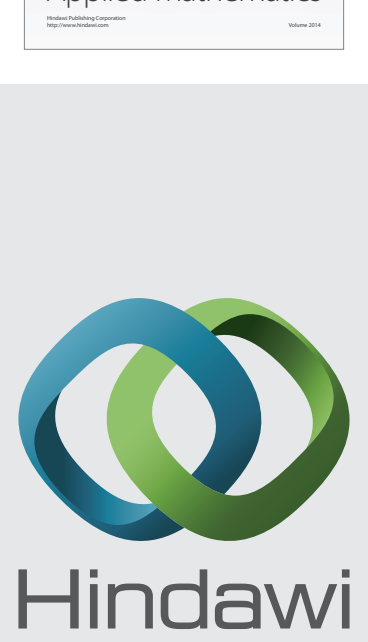

Submit your manuscripts at http://www.hindawi.com
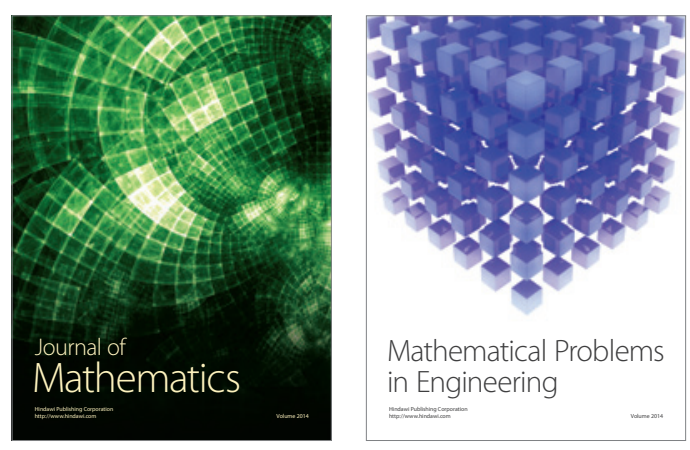

Mathematical Problems in Engineering


Journal of

Function Spaces
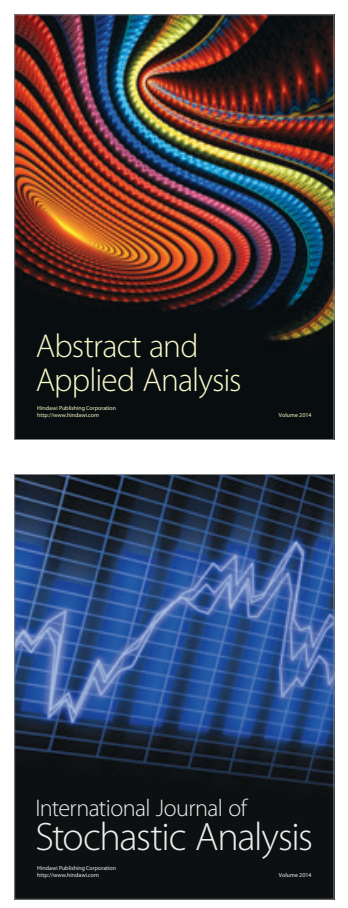

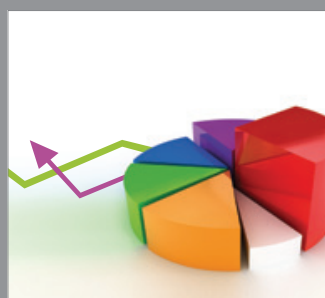

ournal of

Probability and Statistics

Promensencen
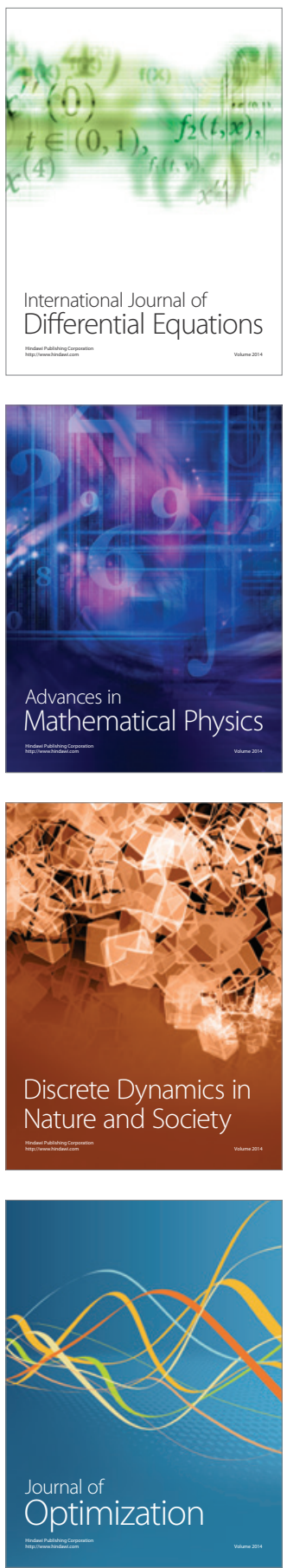